\title{
TOTALLY REAL MINIMAL SUBMANIFOLDS OF A COMPLEX PROJECTIVE SPACE
}

\author{
FRANCISCO URBANO
}

\begin{abstract}
An $n$-dimensional positively curved compact totally real minimal submanifold of an $n$-dimensional complex projective space is totally geodesic.
\end{abstract}

1. Statement of result. Let $C P^{n}$ be an $n$-dimensional complex projective space with the Fubini-Study metric of constant holomorphic sectional curvature $c$, and let $M$ be an $n$-dimensional compact totally real minimal submanifold immersed in $C P^{n}$. We denote by $K$ the sectional curvature of $M$. K. Ogiue [2] proved that if $K>$ $(n-2) c / 4(2 n-1)$, then $M$ is totally geodesic. This result was improved by $\mathrm{B}$. Y. Chen and C. S. Houh [1] who obtained that if $K \geqslant(n-2) c / 4(2 n-1)$, then either $M$ is totally geodesic or $n=2$ and $M$ is a flat surface. This result for $n=2$ was proved by S. T. Yau [3]. In this paper, using methods different from those of [1] and [2], we prove

THEOREM. Let $M$ be an $n$-dimensional compact totally real minimal submanifold immersed in $C P^{n}$. If $K>0$, then $M$ is totally geodesic.

2. Preliminaries. Let $M$ be an $n$-dimensional totally real submanifold immersed in $C P^{n}$. For any vector fields $X$ and $Y$ tangent to $M$, we have

$$
A_{J Y} X=-J \sigma(X, Y), \quad D_{X} J Y=J \nabla_{X} Y,
$$

where $A$ (respectively $\sigma$ ) denotes the Weingarten endomorphism (respectively, the second fundamental form) of $M$ in $C P^{n}, \nabla$ (respectively $D$ ) denotes the Riemannian connection of $M$ (respectively the normal connection of $M$ in $C P^{N}$ ), and $J$ denotes the complex structure of $C P^{n}$.

From (1), and using the equations of Gauss and Ricci, it is easy to obtain

$$
R^{\perp}(X, Y, J Z, J W)=R(X, Y, Z, W)
$$

for any vector fields $X, Y, Z$ and $W$ tangent to $M$, where $R$ and $R^{\perp}$ denote the curvature tensors associated with $\nabla$ and $D$ respectively.

If $\tilde{\nabla} \sigma$ denotes the covariant derivative of $\sigma$, we define the second covariant derivative of $\sigma$ by

$$
\begin{aligned}
\left(\tilde{\nabla}^{2} \boldsymbol{\sigma}\right)(X, Y, Z, W)= & D_{X}((\tilde{\nabla} \boldsymbol{\sigma})(Y, Z, W))-(\tilde{\nabla} \boldsymbol{\sigma})\left(\nabla_{X} Y, Z, W\right) \\
& -(\tilde{\nabla} \boldsymbol{\sigma})\left(Y, \nabla_{X} Z, W\right)-(\tilde{\nabla} \boldsymbol{\sigma})\left(Y, Z, \nabla_{X} W\right) .
\end{aligned}
$$

Received by the editors March 9, 1984.

1980 Mathematics Subject Classification. Primary 53C40. 
It is clear that $\tilde{\nabla} \sigma$ is symmetric, and

$$
\begin{aligned}
\left(\tilde{\nabla}^{2} \sigma\right)(X, Y, Z, W)= & \left(\tilde{\nabla}^{2} \sigma\right)(Y, X, Z, W)+R^{\perp}(X, Y) \sigma(Z, W) \\
& -\sigma(R(X, Y) Z, W)-\sigma(R(X, Y) W, Z) .
\end{aligned}
$$

3. Proof of Theorem. Let $T_{1} M$ be the unit tangent bundle of $M$, and $f: T_{1} M \rightarrow \mathbf{R}$ the function given by $f(v)=\langle\sigma(v, v), J v\rangle$, where $\langle$,$\rangle denotes the metric of C P^{n}$ as well as that of $M$. Since $T_{1} M$ is compact, $f$ attains the maximum at a unit vector $v$ tangent to $M$ at a point $p$. For any unit vector $u$ tangent to $M$ at $p$, let $\alpha(t)=$ $(\gamma(t), V(t))$, let $t \in(-\delta, \delta)$ be a curve in $T_{1} M$ such that $\gamma(t)$ is the only geodesic in $M$ with $\gamma(0)=p$ and $\gamma^{\prime}(0)=u$, and let $V(t)$ be the parallel vector field along $\gamma$ with $V(0)=v$. Then, using (1) we have

$$
0=d f_{V}(u)=(d / d t)_{t=0}\langle\sigma(V(t), V(t)), J V(t)\rangle=\langle(\tilde{\nabla} \sigma)(u, v, v), J v\rangle
$$

and

$$
0 \geqslant d^{2} f_{v}(u, u)=\left\langle\left(\tilde{\nabla}^{2} \sigma\right)(u, u, v, v), J v\right\rangle .
$$

Now, using (1), (2) and (3), we have

$$
\begin{aligned}
d^{2} f_{v}(u, u)= & \left\langle\left(\tilde{\nabla}^{2} \sigma\right)(v, v, u, u), J v\right\rangle \\
& +2 R(u, v, v, J \sigma(u, v))+R(u, v, u, J \sigma(v, v)) .
\end{aligned}
$$

On the other hand, let $S$ be the fiber of $T_{1} M$ over $p$. Then $f / S$ attains the maximum at $v$, and so, if $\beta(t), t \in(-\delta, \delta)$ is a curve in $S$ with $\beta(0)=v$, $\left\|\beta^{\prime}(t)\right\|=1$ and $\beta^{\prime}(0)=u$, we have, using (1),

$$
0=d(f / S)_{v}(u)=(d / d t)_{t=0}\langle\sigma(\beta(t), \beta(t)), J \beta(t)\rangle=3\langle\sigma(v, v), J u\rangle
$$

and

$$
\begin{aligned}
0 & \geqslant d^{2}(f / S)_{v}(u, u)=6\left\langle\sigma\left(\beta^{\prime}(0), v\right), J \beta^{\prime}(0)\right\rangle+3\left\langle\sigma(v, v), J \beta^{\prime \prime}(0)\right\rangle \\
& =6\langle\sigma(u, v), J u\rangle-3\langle\sigma(v, v), J v\rangle=6\langle\sigma(u, u), J v\rangle-3 f(v) .
\end{aligned}
$$

Since (6) is true for any unit vector $u$ orthogonal to $v$, we have $\sigma(v, v)=f(v) J v$, and so, from (1) $v$ is an eigenvector of $A_{J v}$ with eigenvalue $f(v)$. Now, we can choose an orthonormal basis $\left\{u_{1}, \ldots, u_{n}\right\}$ of $T_{p} M$ which diagonalizes $A_{J v}$ such that $u_{n}=v$. If $\rho_{i}, 1 \leqslant i \leqslant n-1$, are the eigenvalues of $u_{i}$, we have from (7)

$$
f(v)-2 \rho_{i} \geqslant 0 .
$$

Now, from (1), (4), (5) and given that $M$ is minimal, we have

$$
0 \geqslant \sum_{i=1}^{n} d^{2} f_{v}\left(u_{i}, u_{i}\right)=\sum_{i=1}^{n-1} K\left(v, u_{i}\right)\left\{f(v)-2 \rho_{i}\right\}
$$

and so from (8), given that $K>0$, it follows that $\rho_{i}=f(v) / 2$ for $1 \leqslant i \leqslant n-1$. Then $0=\operatorname{Trace} A_{J v}=(n+1) f(v) / 2$, which implies $f(v)=0$. Since $f(-u)=$ $-f(u)$, and $v$ is a maximum for $f$, we have $f=0$. Now, in the same way that we used to obtain (6), we have $\sigma=0$.

ACKNowledgement. The author wishes to thank Professor A. Ros for his help. 


\section{REFERENCES}

1. B. Y. Chen and C. S. Houh, Totally real submanifolds of a quaternion projective space, Ann. Mat. Pura Appl. (4) 120 (1979), 185-199.

2. K. Ogiue, Positively curved totally real minimal submanifolds immersed in a complex projective space, Proc. Amer. Math. Soc. 56 (1976), 264-266.

3. S. T. Yau, Submanifolds with constant mean curvature. I, Amer. J. Math. 96 (1974), 346-366.

Departamento de Geometria y Topologia, facultad de Ciencias, Universidad de Granada, Granada, SPAIN 